\title{
TOTAL FORCING SETS AND ZERO FORCING SETS IN TREES
}

\author{
RANDY DAVILA ${ }^{1,2}$ \\ AND \\ Michael A. Henning ${ }^{1}$ \\ ${ }^{1}$ Department of Pure and Applied Mathematics \\ University of Johannesburg \\ Auckland Park 2006, South Africa \\ ${ }^{2}$ Department of Mathematics and Statistics \\ University of Houston-Downtown \\ Houston, TX 77002, USA \\ e-mail: davilar@uhd.edu \\ mahenning@uj.ac.za
}

\begin{abstract}
A dynamic coloring of the vertices of a graph $G$ starts with an initial subset $S$ of colored vertices, with all remaining vertices being non-colored. At each discrete time interval, a colored vertex with exactly one non-colored neighbor forces this non-colored neighbor to be colored. The initial set $S$ is called a forcing set of $G$ if, by iteratively applying the forcing process, every vertex in $G$ becomes colored. If the initial set $S$ has the added property that it induces a subgraph of $G$ without isolated vertices, then $S$ is called a total forcing set in $G$. The minimum cardinality of a total forcing set in $G$ is its total forcing number, denoted $F_{t}(G)$. We prove that if $T$ is a tree of order $n \geq 3$ with maximum degree $\Delta$ and with $n_{1}$ leaves, then $n_{1} \leq F_{t}(T) \leq \frac{1}{\Delta}((\Delta-1) n+1)$. In both lower and upper bounds, we characterize the infinite family of trees achieving equality. Further we show that $F_{t}(T) \geq F(T)+1$, and we characterize the extremal trees for which equality holds.
\end{abstract}

Keywords: forcing set, forcing number, total forcing set, total forcing number.

2010 Mathematics Subject Classification: 05C69. 
[1] AIM Special Work Group, Zero forcing sets and the minimum rank of graphs, Linear Algebra Appl. 428 (2008) 1628-1648.

doi:10.1016/j.laa.2007.10.009

[2] D. Amos, Y. Caro, R. Davila and R. Pepper, Upper bounds on the k-forcing number of a graph, Discrete Appl. Math. 181 (2015) 1-10.

doi:10.1016/j.dam.2014.08.029

[3] F. Barioli, W. Barrett, S.M. Fallat, T. Hall, L. Hogben, B. Shader, P. van den Driessche and H. van der Holst, Parameters related to tree-width, zero forcing, and maximum nullity of a graph, J. Graph Theory 72 (2013) 146-177. doi:10.1002/jgt.21637

[4] D. Burgarth, V. Giovannetti, L. Hogben, S. Severini and M. Young, Logic circuits from zero forcing, Nat. Comput. 14 (2015) 485-490. doi:10.1007/s11047-014-9438-5

[5] B. Brimkov and I.V. Hicks, Complexity and computation of connected zero forcing, Discrete Appl. Math. 229 (2017) 31-45.

doi:10.1016/j.dam.2017.05.016

[6] Y. Caro and R. Pepper, Dynamic approach to k-forcing, Theory Appl. Graphs 2(2) (2015) Article 2.

[7] C. Chekuri and N. Korula, A graph reduction step preserving element-connectivity and applications, Automata, Languages and Programming, Springer (2009) 254-265.

[8] R. Davila, Bounding the Forcing Number of a Graph, Masters Thesis (Rice University, 2015).

[9] R. Davila and M.A. Henning, The forcing number of graphs with a given girth, Quaest. Math. 41 (2018) 189-204. doi:10.2989/16073606.2017.1376230

[10] R. Davila and M.A. Henning, On the total forcing number of a graph, manuscript (2017). arXiv:1702.06035

[11] R. Davila and F. Kenter, Bounds for the zero forcing number of a graph with large girth, Theory Appl. Graphs 2 (2015) Article 1.

[12] C. Edholm, L. Hogben, J. LaGrange and D. Row, Vertex and edge spread of zero forcing number, maximum nullity, and minimum rank of a graph, Linear Algebra Appl. 436 (2012) 4352-4372. doi:10.1016/j.laa.2010.10.015

[13] D.K. Garnick, Y.H. Harris Kwong and F. Lazebnik, Extremal graphs without threecycles or four-cycles, J. Graph Theory 17 (1993) 633-645. doi:10.1002/jgt.3190170511

[14] M. Gentner, L.D. Penso, D. Rautenbach and U.S. Souza, Extremal values and bounds for the zero forcing number, Discrete Appl. Math. 214 (2016) 196-200.

doi:10.1016/j.dam.2016.06.004 
[15] M. Gentner and D. Rautenbach, Some bounds on the zero forcing number of a graph, Discrete Appl. Math. 236 (2018) 203-213.

doi:10.1016/j.dam.2017.11.015

[16] T.W. Haynes, S.T. Hedetniemi and P.J. Slater, Fundamentals of Domination in Graphs (Marcel Decker, Inc., NY, 1998).

[17] T.W. Haynes, S.T. Hedetniemi, S.T. Hedetniemi and M.A. Henning, Domination in graphs applied to electric power networks, SIAM J. Discrete Math. 15 (2002) 519-529. doi: $10.1137 /$ S0895480100375831

[18] M.A. Henning and A. Yeo, Total Domination in Graphs (Springer Monographs in Mathematics, 2013). doi:10.1007/978-1-4614-6525-6

[19] L. Hogben, M. Huynh, N. Kingsley, S. Meyer, S. Walker and M. Young, Propagation time for zero forcing on a graph, Discrete Appl. Math. 160 (2012) 1994-2005. doi:10.1016/j.dam.2012.04.003

[20] L. Lu, B. Wu and Z. Tang, Proof of a conjecture on the zero forcing number of a graph, Discrete Appl. Math. 213 (2016) 233-237. doi:10.1016/j.dam.2016.05.009

[21] O. Ore, Theory of Graphs (Amer. Math. Soc., Providence, RI, 1962). doi: $10.1090 /$ coll $/ 038$

[22] M. Trefois and J.C. Delvenne, Zero forcing number, constrained matchings and strong structural controllability, Linear Algebra Appl. 484 (2015) 199-218. doi:10.1016/j.laa.2015.06.025

Received 28 September 2017

Revised 13 March 2018

Accepted 23 March 2018 